\title{
MicroRNA-30a Suppresses the Activation of Hepatic Stellate Cells by Inhibiting Epithelial-to-Mesenchymal Transition
}

\author{
Jianjian Zheng ${ }^{\mathrm{a}}$ Wei Wang ${ }^{\mathrm{b}, \mathrm{c}}$ Fujun Yu ${ }^{\mathrm{d}}$ Peihong Dong ${ }^{\mathrm{e}}$ Bicheng Chen ${ }^{\mathrm{a}}$ \\ Mengtao Zhou ${ }^{\dagger}$
}

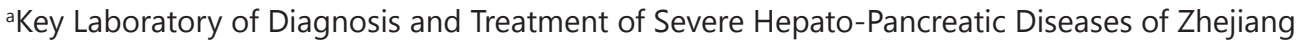
Province, The First Affiliated Hospital of Wenzhou Medical University, Wenzhou, bepartment of Laboratory Medicine, The First Affiliated Hospital of Shenzhen University, Shenzhen, 'Department of Laboratory Medicine, Shenzhen Second People's Hospital, Shenzhen, 'Department of Gastroenterology, The First Affiliated Hospital of Wenzhou Medical University, Wenzhou, eDepartment of Infectious Diseases, The First Affiliated Hospital of Wenzhou Medical University, Wenzhou, fDepartment of Surgery, The First Affiliated Hospital of Wenzhou Medical University, Wenzhou, China

\section{Key Words}

MicroRNA-30a • Epithelial-to-mesenchymal transition • Snai1 • Hepatic stellate cells

\begin{abstract}
Background/Aims: The activation of hepatic stellate cells (HSCs) is considered as a pivotal event in liver fibrosis and epithelial-mesenchymal transition (EMT) process has been reported to be involved in HSC activation. It is known that microRNAs (miRNAs) play a pro-fibrotic or anti-fibrotic role in HSC activation. Recently, emerging studies show that miR-30a is downregulated in human cancers and over-expression of miR-30a inhibits tumor growth and invasion via suppressing EMT process. However, whether miR-30a could regulate EMT process in HSC activation is still unclear. Methods: miR-30a expression was quantified using real-time PCR in carbon tetrachloride $\left(\mathrm{CCl}_{4}\right)$-induced rat liver fibrosis, activated HSCs and patients with cirrhosis. Roles of miR-30a in liver fibrosis in vivo and in vitro were also analyzed. Luciferase activity assays were performed to examine the binding of miR-30a to the 3 '-untranslated region of snail family transcriptional repressor 1 (Snai1). Results: miR-30a was downregulated in human cirrhotic tissues. In $\mathrm{CCl}_{4}$ rats, reduced miR-30a was found in fibrotic liver tissues as well as isolated HSCs. There was a significant reduction in miR-30a in primary HSCs during culture days. miR-30a over-expression resulted in the suppression of $\mathrm{CCl}_{4}$-induced liver fibrosis. Restoration of miR-30a led to the inhibition of HSC activation including cell proliferation, $\alpha-S M A$ and collagen expression. Notably, miR-30a inhibited EMT process, with a reduction in TGF- $\beta 1$ and Vimentin as well as an increase in GFAP and E-cadherin. miR-30a induced a significant reduction in Snail protein expression when compared with the control. Interestingly, Snail protein expression was increased during liver fibrosis, indicating that there
\end{abstract}

J. Zheng, W. Wang and F. Yu contributed equally to this work.

Mengtao Zhou

and Bicheng Chen

KARGER
Department of Surgery, Key Laboratory of Diagnosis and Treatment of Severe Hepato-

Pancreatic Diseases, The First Affiliated Hospital of Wenzhou Medical University (China)

E-Mailzmt0417@hotmail.com, fire717@163.com 


\section{Cellular Physiology Cell Physiol Biochem 2018;46:82-92

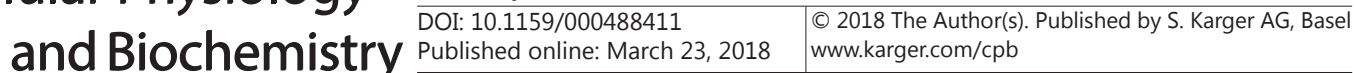 \\ Zheng et al.: MiR-30a Inhibits EMT in HSC Activation by Targeting Snai1}

may be a negative correlation between miR-30a level and Snai1 protein expression. Further studies demonstrated that Snail was a target of miR-30a. Conclusion: Our results suggest that miR-30a inhibits EMT process, at least in part, via reduction of Snai1, leading to the suppression of HSC activation in liver fibrosis.

(C) 2018 The Author(s)

Published by S. Karger AG, Basel

\section{Introduction}

Liver fibrosis, which could be caused by viral hepatitis, alcohol (non-alcoholic) steatohepatitis and several other etiologies persistent damage and stimulation, is a major cause of morbidity and mortality worldwide. With the progression of liver fibrosis, it may further develop into liver cirrhosis and hepatocellular carcinoma, leading to a major public health concern [1]. Liver fibrosis, characterized by accumulated extracellular matrix (ECM) proteins such as type I collagen, may occur in almost all patients with chronic liver diseases [2]. In China, there are far more than 93 million people infected with hepatitis B virus [3]. Therefore, it is urgent to explore an effective treatment for liver fibrosis.

It is well known that activated hepatic stellate cells (HSCs), playing a pivotal role in the development of liver fibrosis, are mainly responsible for the excessive accumulation of ECM proteins in the liver [4]. Generally, HSCs are quiescent in the normal liver. Upon response fibrogenic stimuli, quiescent HSCs will develop into proliferative and fibrogenic, leading to an imbalance between ECM proteins generation and their degradation in liver fibrosis. Recently, increasing evidence shows that the activation of HSCs is considered as an epithelial-mesenchymal transition (EMT) phenomenon, which is defined as the process whereby epithelial cells gradually lose their epithelial signatures, while acquiring the characteristics of mesenchymal cells [5]. Emerging evidence suggests that activated EMT process contributes to HSC trans-differentiation and liver fibrosis. For example, Choi et al. previously found that leptin promotes the myofibroblastic phenotype in hepatic stellate cells by Hedgehog-enhanced EMT process [6]. Our previous studies demonstrated that lncRNA-PVT1 epigenetically down-regulates PTCH1 expression via competitively binding microRNA-152 (miR-152), contributing to EMT process in liver fibrosis [7]. Therefore, inhibiting EMT process in liver fibrosis may be an effective therapeutic strategy.

miRNAs are single stranded, small non-coding RNAs molecules that induce target mRNA degradation and repress mRNA translation by interacting with the 3'-untranslated region (3'-UTR) of target genes [8]. It is known that abnormal expressions of miRNAs could be found in a variety of human diseases such as cancers and liver fibrosis $[9,10]$. Notably, numerous studies have already shown that miRNAs are involved in HSC activation and may act as regulators of HSC activation [11-15]. For instance, Tu et al. found that miR-101 suppresses liver fibrosis by targeting the transforming growth factor-beta (TGF- $\beta$ ) signalling pathway [16].

miR-30a, located on human chromosome 6q.13, is a member of the miR-30 family and has been reported to participate in various cellular processes [17, 18]. A growing body of evidence suggests that miR-30a is down-regulated in fibrotic livers and acts as a key regulator in myocardial fibrosis and peritoneal fibrosis [19-21]. It has been demonstrated that miR30a can ameliorate hepatic fibrosis through suppressing Beclin-mediated autophagy [22]. Increasing evidence shows that miR-30a contributes to the suppression of EMT process in various cancers [23-25]. In this study, we aimed to explore whether miR-30a-mediated EMT process is involved in HSC activation.

\section{Materials and Methods}

\section{Human Specimens}

In this study, 35 healthy controls and 35 liver cirrhosis patients undergoing partial liver resection or liver biopsy were selected from the First Affiliated Hospital of Wenzhou Medical University. Liver cirrhosis 


\section{Cellular Physiology Cell Physiol Biochem 2018;46:82-92 \begin{tabular}{ll|l} 
and Biochemistry Published online: March 23, 2018 & $\begin{array}{l}\text { C) } 2018 \text { The Author(s). Published by S. Karger AG, Basel } \\
\text { www.karger.com/cpb }\end{array}$ \\
\hline
\end{tabular}}

Zheng et al.: MiR-30a Inhibits EMT in HSC Activation by Targeting Snai1

was diagnosed by liver biopsy and/or a typical appearance of the liver on abdominal ultrasound and/or computed tomography scan. Informed consent for use of liver samples was obtained from all participants. The project was approved by the Ethics Committee of the First Affiliated Hospital of Wenzhou Medical University and was performed in compliance with the Declaration of Helsinki.

\section{Isolation and culture of rat HSCs}

Adult male Sprague-Dawley rats (body weight, 400-500 g) were used for HSC isolation as described previously [26]. Cells were cultured in Dulbecco's modified Eagle's medium supplemented with 10\% fetal bovine serum, $100 \mathrm{U} / \mathrm{ml}$ penicillin and $100 \mathrm{U} / \mathrm{ml}$ streptomycin. The purity of cultures was confirmed by immunocytochemical staining for $\alpha$-smooth muscle actin ( $\alpha$-SMA) and the purity reached $>98 \%$. The harvested primary HSCs were studied at day 0 after isolation throughout all the studies. Primary HSCs at Day 0 were transfected with miR-30a mimics or the negative control (miR-NC) for $48 \mathrm{~h}$ using Lipofectamine RNAiMAX at a final concentration of $10 \mathrm{nM}$.

\section{Carbon tetrachloride $\left(\mathrm{CCl}_{4}\right)$ liver injury model}

Adult male Sprague-Dawley (180-220 g) rats were provided by the Experimental Animal Center of Wenzhou Medical University. Liver fibrosis was induced by administration of $2 \mathrm{ml}$ of $\mathrm{CCl}_{4} /$ olive oil $(1: 1, \mathrm{v} / \mathrm{v}) /$ $\mathrm{kg}$ body weight by intraperitoneal injection twice weekly for up to 6 weeks [27]. Rats were randomly divided into four groups, including olive oil $(n=6), \mathrm{CCl}_{4}(\mathrm{n}=6), \mathrm{CCl}_{4}$ plus the lentiviral vector containing negative control (Lenti-NC) $(\mathrm{n}=6)$ and $\mathrm{CCl}_{4}$ plus lentiviral miR-30a mimics (Lenti-miR-30a) $(\mathrm{n}=6)$, respectively. LentimiR-30a and Lenti-NC were obtained from Shanghai GeneChem. Lenti-miR-30a or Lenti-NC was injected via the tail vein only once at three weeks after $\mathrm{CCl}_{4}$ injection $\left(1 \times 10^{9}\right.$ transducing unit/100 $\left.\mu \mathrm{l}\right)$. After the following 3-weeks $\mathrm{CCl}_{4}$ treatment, the rats were sacrificed. The experimental protocol was approved by the Institutional Animal Committee of Wenzhou Medical University. All animals received care in accordance with 'Guide for the Care and Use of Laboratory Animals'. All rats were sacrificed under anesthesia at the end of six weeks and the livers were removed for further analysis. The liver tissues were used for Masson staining by fixation with $10 \%$ formalin. Quantitative analysis for the Masson-positive area was calculated from five fields for each liver slice.

\section{Immunohistochemistry and Serum ALT analysis}

Immunohistochemical staining was performed on the sections ( $3 \mu \mathrm{m}$ thick) from the liver tissues, as described previously [28]. Briefly, after deparaffinization, hydration, and antigen retrieval, samples were incubated overnight at $4^{\circ} \mathrm{C}$ with a primary antibody against $\alpha$-SMA $(1: 100)$ and then with a biotinylated secondary antibody. $\alpha$-SMA expression was visualized by 3 , 3'-diaminobenzidine tetrahydrochloride (DAB) staining. Slides were counterstained with hematoxylin before dehydration and mounting $\alpha$-SMA-positive areas within the fibrotic region were then observed. Quantitative analysis was calculated from five fields for each liver slice. Serum ALT level (Sigma-Aldrich, cat\# MAK052) was according to the manufacturer's protocols.

\section{Transwell migration assay}

Cells were placed in the top chamber of transwell migration chambers $(8 \mu \mathrm{m}$; Millipore, Billerica, MA, USA). After $48 \mathrm{~h}$, cells which had not migrated to the lower chamber were removed from the upper surface of the transwell membrane with a cotton swab. Migrating cells on the lower membrane surface were fixed, stained, photographed and counted using a microscope at $\times 100$. Experiments were assayed in triplicate, and $\geq 5$ fields were counted in each experiment.

\section{Proliferation assay}

Cell proliferation was assessed using CCK-8 (Dojindo, Kumamoto, Japan) according to manufacturer's instructions. Cells were seeded in 96-well plates at a density of $1 \times 10^{3}$ cells per well and cultured for $24 \mathrm{~h}$. Next, cells were transfected with miR-30a mimics. After $48 \mathrm{~h}$, CCK-8 solution was added to each well in the 96-well plates. Then, cells were incubated for additional $2 \mathrm{~h}$ at $37^{\circ} \mathrm{C}$. Absorbance was determined at 450 $\mathrm{nm}$ on a microplate reader (Molecular Devices, Sunnyvale, CA, USA). Furthermore, HSCs were labelled with EdU for $12 \mathrm{~h}$. HSC proliferative rate was detected using a Cell-Light ${ }^{\mathrm{TM}}$ EdU In vitro Imaging Detection Kit (Guangzhou RiboBio Co., Ltd., cat\# C10310-1) according to the manufacturer's instructions. 


\section{Cellular Physiology Cell Physiol Biochem 2018;46:82-92 \begin{tabular}{ll|l} 
and Biochemistry & $\begin{array}{l}\text { DOI: 10.1159/000488411 } \\
\text { Published online: March 23, } 2018\end{array}$ & $\begin{array}{l}\text { (c) } 2018 \text { The Author(s). Published by S. Karger AG, Basel } \\
\text { www.karger.com/cpb }\end{array}$
\end{tabular}

\section{Quantitative real-time PCR ( $q R T-P C R)$}

Total RNA was extracted from liver tissues and cells using miRNeasy Mini kit (Qiagen, Valencia, CA, USA) according to manufacturer's instructions. Gene expression was measured by qRT-PCR using SYBR Green real-time PCR Master Mix (Toyobo, Osaka, Japan), and a set of gene-specific oligonucleotide primers (snail family transcriptional repressor 1 (Snai1): forward 5'-GCAGAGTTGTCTACCGACC T-3', reverse 5'-AGGTGAACTCCACACACGC-3'; E-cadherin: forward 5'-CCAC CAGATGACGATACCCG-3', reverse 5'-GAATCACTTCCGGTCTGGCA-3'; Vimentin: forward 5'-TCCTTCGAAGCCATGTCCAC-3' ${ }^{\prime}$, reverse 5'-GTGGTC ACATAGCTCCGGTT-3'; TGF- $\beta 1$ : forward $5^{\prime}$-GACTCTCCACCTGCAAGACC-3', reverse $5^{\prime}$-GGACTGGC GAGCCTTAGTTT-3'; glial fibrillary acidic protein (GFAP): forward $5^{\prime}$-TTGACCTGCGACCTTGAGTC-3' ${ }^{\prime}$, reverse 5'-GAGTGCCTCCT GGTAACTCG-3'. In addition, the primers of alpha-1 (I) collagen (Col1A1), $\alpha$-SMA, GAPDH and U6 were designed as described previously [29]. miR-30a expression was detected using the TaqMan MicroRNA Assay (Applied Biosystems, Foster City, CA). The GAPDH and U6 levels were used to normalize the relative abundance of mRNAs and miR-30a, respectively. The expression levels $\left(2^{-\Delta \Delta \mathrm{Ct}}\right)$ of mRNAs and miR-30a were calculated as described previously [23].

\section{Western blot analysis}

Equal amounts of protein were subjected to sodium dodecy1 sulphate-polyacrylamide gel electrophoresis and then transferred onto membranes. The membrane was incubated with primary antibodies overnight at $4^{\circ} \mathrm{C}$, then secondary IRdye800-conjugated goat anti-mouse IgG or goat anti-rabbit IgG at $37^{\circ} \mathrm{C}$ for $1 \mathrm{~h}$. Antibody binding was detected using an Odyssey infrared scanner (Li-Cor Biosciences Inc., Lincoln, NE, USA).

\section{Luciferase activity assay}

HEK293T cells were co-transfected with either luciferase reporter plasmid harboring wild-type Snai1 3'UTR (pmirGLO-Snai1-Wt) or mutant Snai1 3'UTR (pmirGLO-Snai1-Mut) together with miR-30a mimics or miR-NC. The 3'UTR of Snai1 was cloned downstream of the firefly luciferase gene. Whether the activity of firefly luciferase is down-regulated or not is depended on the interaction between miR-30a and the 3'UTR of Snai 1 mRNA. Approximately $48 \mathrm{~h}$ after transfection, the cells were harvested and luciferase activity was determined by a luminometer using a Dual-Luciferase Reporter Assay System (Promega, Madison, WI, USA).

\section{Statistical analysis}

Data from at least three independent experiments were expressed as the mean \pm SD. Differences between multiple groups were evaluated using one-way analysis of variance. Differences between two groups were compared using a Student's $t$-test. $P<0.05$ was considered significant. All statistical analyses were performed with SPSS software (version 13; SPSS, Chicago, IL).

\section{Results}

\section{Down-regulation of miR-30a in liver fibrosis}

To gain insights into the possible involvement of miR-30a in liver fibrosis, miR-30a expression was firstly detected in liver tissues from patients with cirrhosis and healthy controls. Compared with healthy liver tissues, miR30a expression was obviously reduced in cirrhotic tissues (Fig. 1A). Primary HSCs were iso-

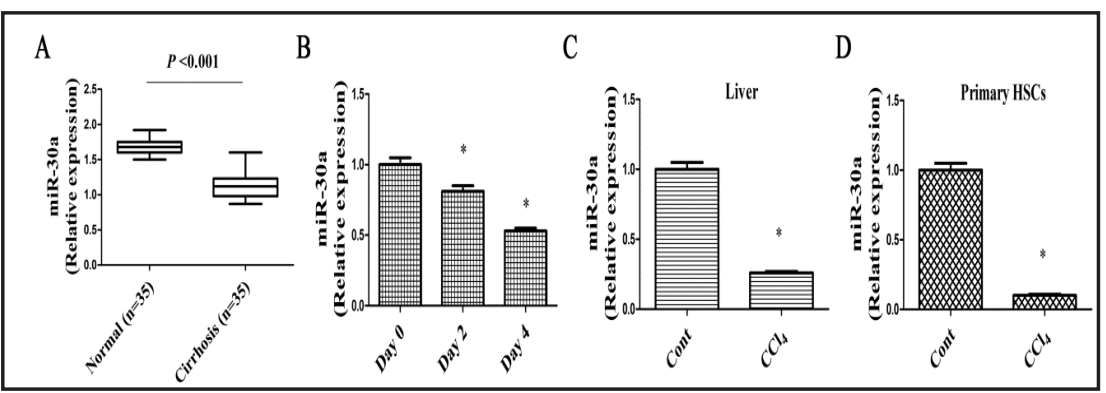

Fig. 1. miR-30a expression is reduced in liver fibrosis. (A) miR-30a in healthy controls and patients with cirrhosis. (B) miR-30a in primary HSCs isolated from healthy rats at Day 0, Day 2 and Day 4. miR-30a in the livers of $\mathrm{CCl}_{4}$ rats (C) and primary HSCs isolated from $\mathrm{CCl}_{4}$ rats (D). ${ }^{*} \mathrm{P}<0.05$ compared to the control. 


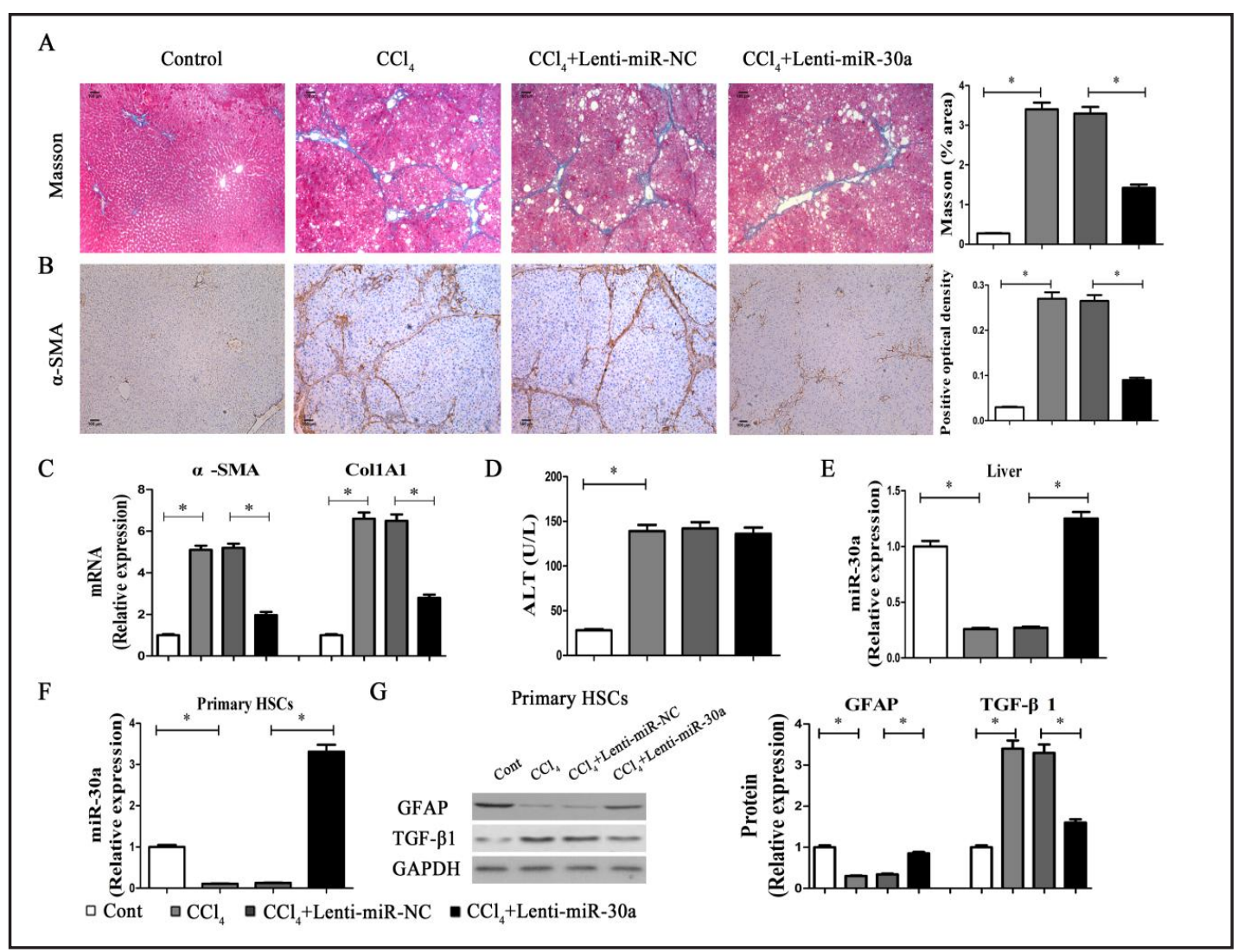

Fig. 2. Restoring of miR-30a inhibits the progression of liver fibrosis in $\mathrm{CCl}_{4}$ rats. (A and B) Collagen expression and $\alpha$-SMA level were assessed by Masson staining and immunohistochemistry, respectively. Scale bar, $100 \mu \mathrm{m}$. (C) The mRNA levels of $\alpha$-SMA and Col1A1. (D) ALT value. (E) miR-30a in the livers of $\mathrm{CCl}_{4}$ rats with miR-30a over-expression and (F) in primary HSCs isolated from $\mathrm{CCl}_{4}$ rats with miR-30a overexpression. (G) The protein levels of GFAP and TGF- $\beta 1$ were analyzed in primary $\mathrm{HSC}$ s isolated from $\mathrm{CCl}_{4}$ rats with miR-30a over-expression. GAPDH was used as internal control. ${ }^{*} \mathrm{P}<0.05$.

lated from the livers of healthy rats and cultured for up to 4 days. Generally, isolated primary HSCs cultured on plastic will gradually become an activated myofibroblastic phenotype during culture days. Next, miR-30a expression was detected in primary rat HSCs during culture days. With time in culture, our results showed a significant reduction in miR-30 level in primary HSCs (Fig. 1B). miR-30a expression was additionally examined in $\mathrm{CCl}_{4}$-induced rat liver fibrosis model. In comparison with the control, miR-30a was down-regulated in the livers of $\mathrm{CCl}_{4}$ rats (Fig. 1C). In line with it, down-regulation of miR-30a was found in primary HSCs isolated from $\mathrm{CCl}_{4}$ rats (Fig. 1D). These data suggest that miR-30a is down-regulated during liver fibrosis.

\section{Restoration of miR-30a ameliorates liver fibrosis in vivo and in vitro}

To investigate the biological roles of miR-30a in liver fibrosis, the effects of miR-30a overexpression on $\mathrm{CCl}_{4}$-induced liver fibrosis were explored. The results of Masson staining indicated that accumulated collagen expression induced by $\mathrm{CCl}_{4}$ was inhibited by miR-30a (Fig. $2 \mathrm{~A}$ ). As shown by immunohistochemical images, $\mathrm{CCl}_{4}$ - enhanced $\alpha$-SMA by was suppressed by miR-30a (Fig. 2B). Consistent with these, the mRNA levels of $\alpha$-SMA and Col1A1 induced by $\mathrm{CCl}_{4}$ were blocked down by miR-30a (Fig. 2C). Interestingly, miR-30a had no effect on ALT value (Fig. 2D). In $\mathrm{CCl}_{4}$ rats, miR-30a over-expression led to a significant increase in miR-30a in the livers as well as primary HSCs (Fig. 2E and Fig. 2F). Notably, in isolated HSCs from $\mathrm{CCl}_{4}$ rats with miR-30a over-expression, miR-30a resulted in an increase in GFAP (epithelial mark- 
Fig. 3. Inhibitory effects of miR-30a on HSC activation in vitro. Primary HSCs at Day 0 were transfected with miR-30a mimics for $48 \mathrm{~h}$ using Lipofectamine RNAiMAX. (A) miR-30a in cells with miR-30a over-expression. Cell proliferation was analyzed by CCK8 assays (B) or EdU assays (C). The mRNA (D) and protein (E) levels of $\alpha$-SMA and CoI1A1 in cells with miR-30a over-expression. ${ }^{*} \mathrm{P}<0.05$.
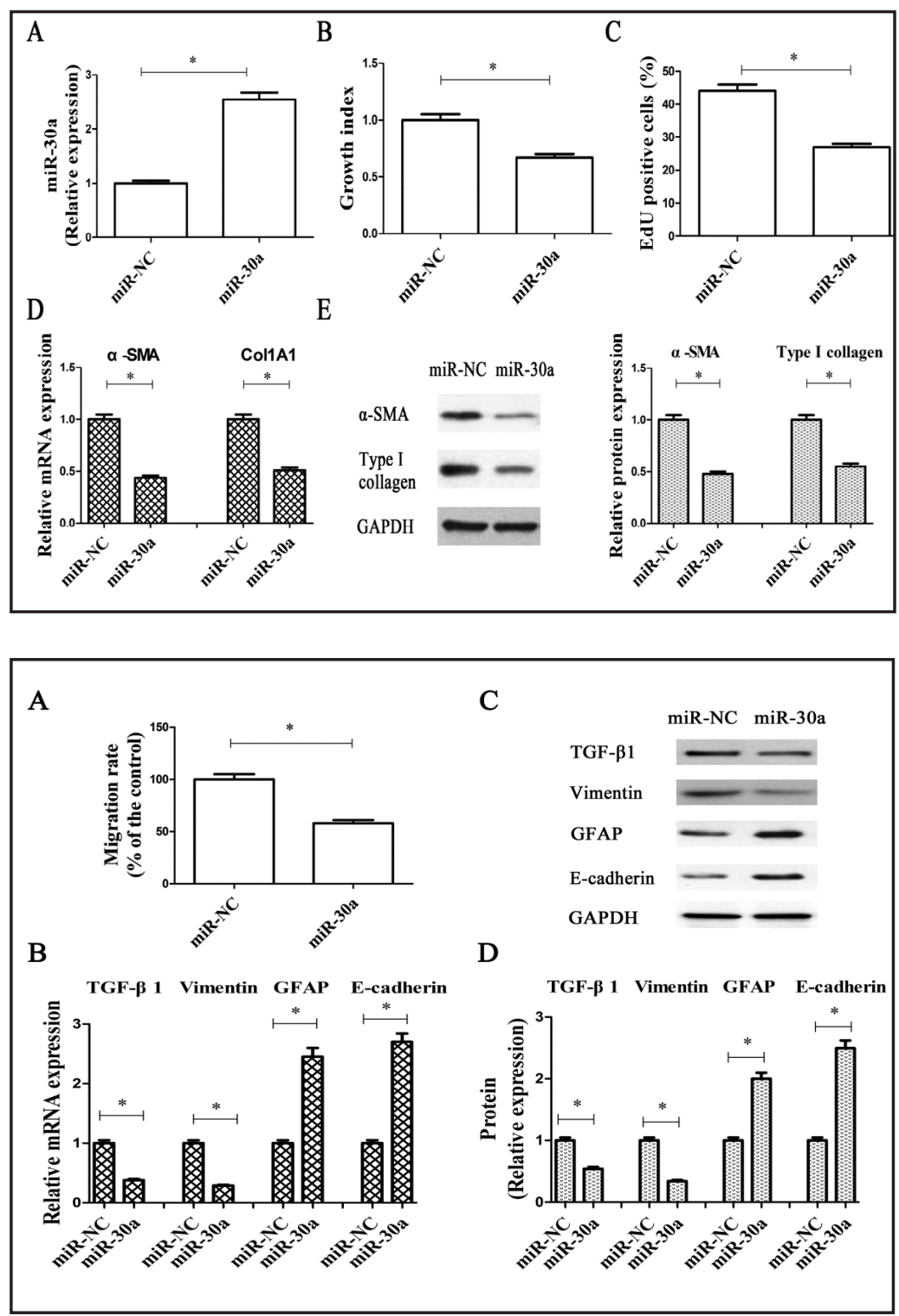

Fig. 4. Inhibitory effects of miR-30a on EMT process. (A) Cell migration was analyzed by transwell migration assay. Five fields of migrated cells in the lower side of transwell were counted with a microscope at $\times 100$. The mRNA (B) and protein ( $C$ and D) levels of E-cadherin, Vimentin, TGF- $\beta 1$ and GFAP were analyzed by qRT-PCR and western blot, respectively. GAPDH was used as internal control. ${ }^{*} \mathrm{P}<0.05$.

\section{in miR-30a (Fig. 3A)}

As shown by CCK8 assays, our results indicated that compared with the control, miR-30a resulted in a significant reduction in cell proliferation (Fig. 3B). EdU assays additionally confirmed an inhibitory role of miR-30a in cell proliferation in primary HSCs (Fig. 3C). Moreover, we assessed the effects of miR-30a on $\alpha$-SMA and collagen expression. miR-30a led to a reduction in the mRNA levels of $\alpha$-SMA and Col1A1 in miR-30a-over-expressing cells (Fig. 3D). Accordingly, immunoblot analysis demonstrated that miR-30a over-expression contributed to the suppression of the proteins of $\alpha$-SMA and type I collagen (Fig. 3E). Our results suggest that miR-30a contributes to the suppression of HSC activation. 
Fig. 5. $\mathrm{miR}-30 \mathrm{a}$ targets Snai1. (A) miR-30a-binding sites in the $3^{\prime} \mathrm{UTR}$ of Snai1 mRNA based on miRDB software. (B) Relative luciferase activities of luciferase reporters bearing wild-type or mutant Snai1 were analyzed $48 \mathrm{~h}$ following transfection with the indicated miR-30a mimics or miR-NC in HEK293T cells. The mRNA (C) and protein (D) expression level of Snai1 in cells with miR-30a over-expression. (E) Immunoblot analysis of Snai1 protein expression in healthy controls $(n=35)$ and patients with cirrhosis $(n=35)$. Immunoblot images of three representative samples in control or cirrhosis group were provided. (F)

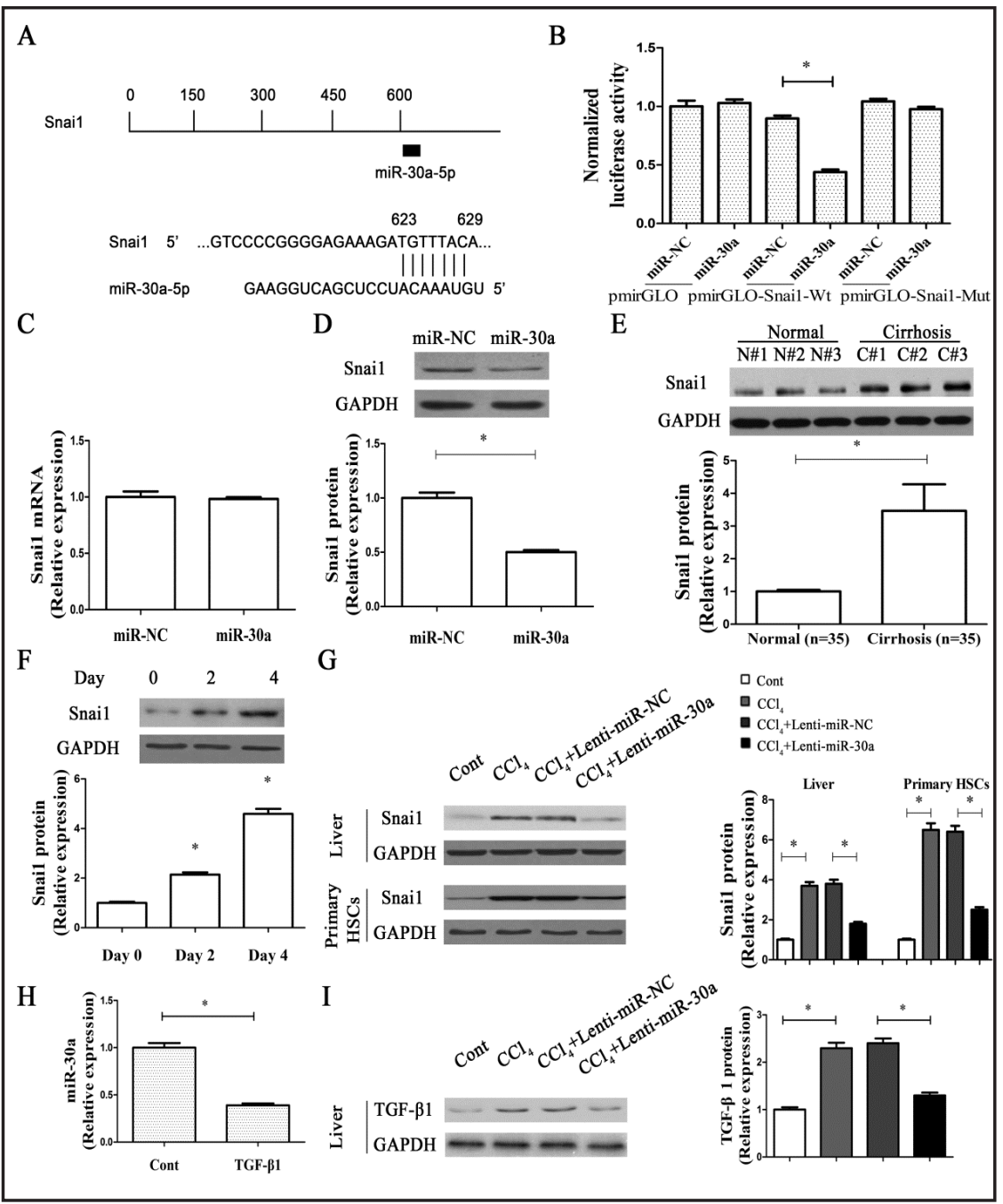

Snai1 protein ex-

pression in primary HSCs at Day 0, Day 2 and Day 4. (G) Snai1 protein expression in the liver of $\mathrm{CCl}_{4}$ rats with miR-30a over-expression and primary HSCs isolated from $\mathrm{CCl}_{4}$ rats with miR-30a over-expression. $(\mathrm{H})$ miR-30a level was detected in primary HSCs after $2 \mathrm{ng} / \mathrm{ml}$ TGF- $\beta 1$ treatment for $48 \mathrm{~h}$. (I) TGF- $\beta 1$ protein expression in the liver of $\mathrm{CCl}_{4}$ rats with miR-30a over-expression. GAPDH was used as internal control. ${ }^{*} \mathrm{P}<0.05$ compared to the control.

miR-30a over-expression inhibits HSC activation through EMT process

Further studies showed that down-regulated HSC migration was found in cells with miR-30a over-expression (Fig. 4A). Next, we explored whether EMT process was involved in the effects of miR-30a on HSC activation. Our results indicated that miR-30a led to a reduction in TGF- $\beta 1$ mRNA and an increase in GFAP mRNA (Fig. 4B). Consistent with the mRNA results, reduced TGF- $\beta 1$ and increased GFAP were found in miR-30a-over-expressing cells, as demonstrated by immunoblot analysis (Fig. 4C and Fig. 4D). In addition, E-cadherin (epithelial marker) and Vimentin (myofibroblastic marker) was examined in miR-30aoverexpressiong cells. Restoration of miR-30a led to an increase in E-cadherin as well as a reduction in Vimentin (Fig. 4B-4D). These data demonstrate that miR-30a suppresses EMT process in HSC activation. 


\section{Cellular Physiology Cell Physiol Biochem 2018:46:82-92

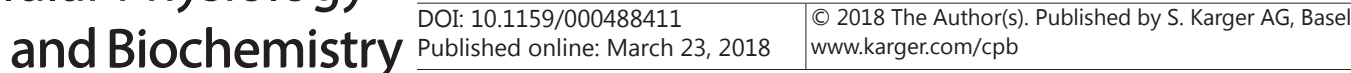

miR-30a inhibits EMT transition through targeting of Snai1

To explore the molecular mechanism of miR-30a in regulation of EMT process during HSC activation, bioinformatic analysis (miRDB) was employed to identify the potential targets of miR-30a. Notably, Snai1, a known transcriptional repressor of E-cadherin and modulator of EMT process, was predicted as a putative target of miR-30a (Fig. 5A). Next, the 3'UTR target sequence of Snai1 mRNA was cloned into the pmirGLO plasmid to confirm whether miR-30a could directly regulate Snai1 expression via the predicted binding site. As shown in Fig. 5B, miR-30a mimics resulted in a significant reduction in luciferase activity of pmirGLOSnai1-Wt. Meanwhile, miR-30a mimics had no effect on the luciferase activity of pmirGLOSnai1-Mut (Fig. 5B). Therefore, Snai1 is a target of miR-30a. Moreover, the effects of miR30 a on Snai1 expression were examined in vitro. miR-30a induced a significant reduction in Snai1 protein with no effect on Snai1 mRNA, indicating that Snai1 is post-transcriptionally regulated by miR-30a (Fig. 5C and Fig. 5D). Further studies were performed to detect Snai1 protein expression during liver fibrosis in vivo and in vitro. Snai1 protein in the liver tissues from patients with cirrhosis was higher than that of healthy controls (Fig. 5E). Also, there was a significant increase in Snai1 protein in primary HSCs during culture days (Fig. 5F). Our previous results demonstrated that miR-30a was down-regulated in liver fibrosis, indicating that there was a negative correlation between miR-30a and Snai1 protein. Consistent with these, Snai1 protein was increased in the livers of $\mathrm{CCl}_{4}$ rats, which was inhibited by miR-30a (Fig. 5G). In primary HSCs isolated from $\mathrm{CCl}_{4}$ rats, miR-30a also contributed to the suppression of $\mathrm{CCl}_{4}$-induced Snai1 protein (Fig. $5 \mathrm{G}$ ). TGF- $\beta 1$ could promote EMT by stimulating Snai1 and suppressing TGF- $\beta 1$-induced Snai1 may contribute to inhibit EMT process [31]. Next, the relation between TGF- $\beta 1$ and miR-30a was examined. We found that TGF- $\beta 1$ treatment induced a significant reduction in miR-30a (Fig. $5 \mathrm{H}$ ). In vitro, miR-30a resulted in the suppression of TGF- $\beta 1$ expression (Fig. 4B-4D). In addition, miR-30a led to the suppression of TGF- $\beta 1$ in the livers of $\mathrm{CCl}_{4}$ rats as well as in primary HSCs isolated from $\mathrm{CCl}_{4}$ rats (Fig. 2G and Fig. 5I). Taken together, all these results suggest that EMT transition could be blocked down by miR-30a, at least in part, through Snai1.

\section{Discussion}

At the present study, our results showed that miR-30a was down-regulated in activated HSCs as well as fibrotic liver tissues. In vitro, restoration of miR-30a contributes to the suppression of HSC activation including the reduction of cell proliferation, $\alpha$-SMA and type I collagen. Similarly, miR-30a ameliorates $\mathrm{CCl}_{4}$-induced rat liver fibrosis, indicating an inhibitory role of miR-30a in liver fibrosis. Notably, EMT process was involved in the effects of miR-30a in HSC activation. Due to restoration of miR-30a, EMT process was inhibited in miR-30a-overexressing cells, with a reduction in TGF- $\beta 1$ and Vimentin as well as an increase in GFAP and E-cadherin. Moreover, HSC migration was significantly reduced by miR-30a. We demonstrate that miR-30a could regulate EMT process in HSC activation and this is a first report.

miR-30a, described as a tumor suppressor, is generally down-regulated in cancers. For example, up-regulation of miR-30a inhibits liver cancer cell proliferation and induces apoptosis through MTDH/PTEN/AKT pathway [32]. A recent study confirms that miR-30a functions as a tumor suppressor in gastric cancer via targeting COX-2 and BCL9 [33]. In this study, miR-30a was reduced in liver fibrosis, similar with the results in cancers [32, 33]. Recently, Chen et al. found that miR-30a over-expression results in the suppression of liver fibrosis by inhibiting Beclin1-mediated autophagy [22]. Our results indicated that miR-30a acts as a suppressive factor in HSC activation, which was consistent with the previous study [22]. Of note, we demonstrate that miR-30a-mediated EMT process is involved in the antifibrotic effects of miR-30a in HSC activation.

Increasing evidence demonstrates that restoration of miR-30a contributes to the suppression of EMT process in various cancers and fibrotic diseases [24, 34, 35], indicating 


\section{Cellular Physiology Cell Physiol Biochem 2018;46:82-92

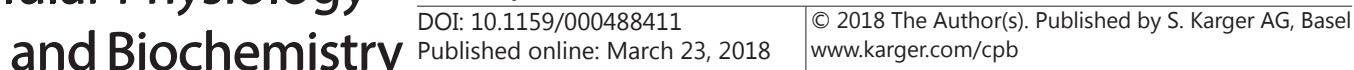 \\ Zheng et al.: MiR-30a Inhibits EMT in HSC Activation by Targeting Snai1}

an inhibitory role of miR-30a in EMT. Generally, TGF- $\beta 1$, Vimentin, E-cadherin and GFAP are considered to be important EMT markers. Many EMT inducers, including transcription factors such as Snai1, Snai2 and ZEB1, have been reported to function via suppressing E-cadherin [36]. In addition to E-cadherin repression, Snai 1 also down-regulates other epithelial markers and up-regulates mesenchymal markers $[36,37]$. In this study, we found that Snai1 is a target of miR-30a, as confirmed by luciferase reporter assays. Our results also demonstrated that miR-30a suppressed Snai1 protein in vitro and in vivo. Accordingly, miR-30a suppressed EMT process in vitro and in vivo. Combined these, miR-30a-mediated EMT process may be through Snai1. It was found that miR-30a was down-regulated in liver fibrosis, whereas Snai1 protein expression was up-regulated. There was a negative correlation between miR$30 \mathrm{a}$ level and Snai1 protein expression. Due to the reason that TGF- $\beta 1$ can promote EMT by stimulating Snai1, the effect of miR-30a on TGF- $\beta 1$ was explored. miR-30a was found to suppress TGF- $\beta 1$ expression in liver fibrosis, suggesting that TGF- $\beta 1$-enhanced Snai1 may be also inhibited by miR-30a. Interestingly, TGF- $\beta 1$ treatment attenuated the level of miR-30a in HSCs. These data further confirm that miR-30a is down-regulated in liver fibrosis. Taken together, miR-30a inhibits EMT process in HSC activation, at least in part, via targeting Snai1.

\section{Conclusion}

The present study suggests that up-regulation of miR-30a suppresses EMT process, at least in part, through inhibiting Snai1, contributing to the suppression of HSC activation in liver fibrosis.

\section{Acknowledgements}

The project was supported by the National Natural Science Foundation of China (No. 81500458/H0317), Zhejiang Provincial Natural Science Foundation of China (No. LY16H030012) and Wenzhou Municipal Science and technology Bureau (No. Y20150091).

\section{Disclosure Statement}

No conflict of interests exists.

\section{References}

1 Popov Y, Schuppan D: Targeting liver fibrosis: strategies for development and validation of antifibrotic therapies. Hepatology 2009;50:1294-1306.

-2 Wang J, Chu ES, Chen HY, Man K, Go MY, Huang XR, Lan HY, Sung JJ, Yu J: microRNA-29b prevents liver fibrosis by attenuating hepatic stellate cell activation and inducing apoptosis through targeting PI3K/AKT pathway. Oncotarget 2015;6:7325-7338.

3 Liu X, Wan X, Li Z, Lin C, Zhan Y, Lu X: Golgi protein 73(GP73), a useful serum marker in liver diseases. Clin Chem Lab Med 2011;49:1311-1316.

-4 Xiao Y, Qu C, Ge W, Wang B, Wu J, Xu L, Chen Y: Depletion of thymosin beta4 promotes the proliferation, migration, and activation of human hepatic stellate cells. Cell Physiol Biochem 2014;34:356-367.

5 Lee JM, Dedhar S, Kalluri R, Thompson EW: The epithelial-mesenchymal transition: new insights in signaling, development, and disease. J Cell Biol 2006;172:973-981.

-6 Choi SS, Syn WK, Karaca GF, Omenetti A, Moylan CA, Witek RP, Agboola KM, Jung Y, Michelotti GA, Diehl AM: Leptin promotes the myofibroblastic phenotype in hepatic stellate cells by activating the hedgehog pathway. J Biol Chem 2010;285:36551-36560. 


\section{Cellular Physiology Cell Physiol Biochem 2018;46:82-92 \begin{tabular}{l|l} 
and Biochemistry & DOI: 10.1159/000488411 \\
Published 2018 The Author(s). Published by S. Karger AG, Basel \\
www.karger.com/cpb
\end{tabular}

7 Zheng J, Yu F, Dong P, Wu L, Zhang Y, Hu Y, Zheng L: Long non-coding RNA PVT1 activates hepatic stellate cells through competitively binding microRNA-152 Oncotarget 2016;7:62886-62897.

8 Bartel DP: MicroRNAs: target recognition and regulatory functions. Cell 2009;136:215-233.

9 Yu F, Lu Z, Chen B, Dong P, Zheng J: microRNA-150: a promising novel biomarker for hepatitis B virusrelated hepatocellular carcinoma. Diagn Pathol 2015;10:129.

10 Zou Y, Cai Y, Lu D, Zhou Y, Yao Q, Zhang S: MicroRNA-146a-5p attenuates liver fibrosis by suppressing profibrogenic effects of TGFbeta1 and lipopolysaccharide. Cell Signal 2017;39:1-8.

11 Yu F, Guo Y, Chen B, Dong P, Zheng J: MicroRNA-17-5p activates hepatic stellate cells through targeting of Smad7 Lab Invest 2015;95:781-789.

12 Yu F, Chen B, Fan X, Li G, Dong P, Zheng J: Epigenetically-Regulated MicroRNA-9-5p Suppresses the Activation of Hepatic Stellate Cells via TGFBR1 and TGFBR2 Cell Physiol Biochem 2017;43:2242-2252.

13 Yu F, Guo Y, Chen B, Shi L, Dong P, Zhou M, Zheng J: LincRNA-p21 Inhibits the Wnt/beta-Catenin Pathway in Activated Hepatic Stellate Cells via Sponging MicroRNA-17-5p. Cell Physiol Biochem 2017;41:1970-1980.

14 Yu F, Yang J, Huang K, Pan X, Chen B, Dong P, Zheng J: The Epigenetically-Regulated microRNA-378a Targets TGF-beta2 in TGF-beta1-Treated Hepatic Stellate Cells. Cell Physiol Biochem 2016;40:183-194.

15 Yu F, Fan X, Chen B, Dong P, Zheng J: Activation of Hepatic Stellate Cells is Inhibited by microRNA-378a-3p via Wnt10a. Cell Physiol Biochem 2016;39:2409-2420.

16 Tu X, Zhang H, Zhang J, Zhao S, Zheng X, Zhang Z, Zhu J, Chen J, Dong L, Zang Y: MicroRNA-101 suppresses liver fibrosis by targeting the TGFbeta signalling pathway. J Pathol 2014;234:46-59.

17 Rodriguez A, Griffiths-Jones S, Ashurst JL, Bradley A: Identification of mammalian microRNA host genes and transcription units. Genome Res 2004;14:1902-1910.

$>18$ Liu L, Meng T, Wang QS, Jin HZ, Sun ZQ Jin B, Fang F, Wang HJ: Association of Beclin-1 and microRNA-30a expression with the severity and treatment response of colorectal cancer. Genet Mol Res 2016;15

-19 Du J, Niu X, Wang Y, Kong L, Wang R, Zhang Y, Zhao S, Nan Y: MiR-146a-5p suppresses activation and proliferation of hepatic stellate cells in nonalcoholic fibrosing steatohepatitis through directly targeting Wnt1 and Wnt5a. Sci Rep 2015;5:16163.

20 Yuan CT, Li XX, Cheng QJ, Wang YH, Wang JH, Liu CL: MiR-30a regulates the atrial fibrillation-induced myocardial fibrosis by targeting snail 1 Int J Clin Exp Pathol 2015;8:15527-15536.

-21 Zhou Q, Yang M, Lan H, Yu X: miR-30a negatively regulates TGF-beta1-induced epithelial-mesenchymal transition and peritoneal fibrosis by targeting Snai1 Am J Pathol 2013;183:808-819.

22 Chen J, Yu Y, Li S, Liu Y, Zhou S, Cao S, Yin J, Li G: MicroRNA-30a ameliorates hepatic fibrosis by inhibiting Beclin1-mediated autophagy. J Cell Mol Med 2017

23 Wang LL, Zhang XH, Zhang X, Chu JK: MiR-30a increases cisplatin sensitivity of gastric cancer cells through suppressing epithelial-to-mesenchymal transition (EMT). Eur Rev Med Pharmacol Sci 2016;20:1733-1739.

24 Park YR, Kim SL, Lee MR, Seo SY, Lee JH, Kim SH, Kim IH, Lee SO, Lee ST, Kim SW: MicroRNA-30a-5p (miR30a) regulates cell motility and EMT by directly targeting oncogenic TM4SF1 in colorectal cancer. J Cancer Res Clin Oncol 2017

25 Chang CW, Yu JC, Hsieh YH, Yao CC, Chao JI, Chen PM, Hsieh HY, Hsiung CN, Chu HW, Shen CY, Cheng CW: MicroRNA-30a increases tight junction protein expression to suppress the epithelial-mesenchymal transition and metastasis by targeting Slug in breast cancer. Oncotarget 2016;7:16462-16478.

-26 Weiskirchen R, Gressner AM: Isolation and culture of hepatic stellate cells. Methods Mol Med 2005;117:99113.

27 Yao QY, Xu BL, Wang JY, Liu HC, Zhang SC, Tu CT: Inhibition by curcumin of multiple sites of the transforming growth factor-beta1 signalling pathway ameliorates the progression of liver fibrosis induced by carbon tetrachloride in rats. BMC Complement Altern Med 2012;12:156.

28 Yu F, Chen B, Dong P, Zheng J: HOTAIR Epigenetically Modulates PTEN Expression via MicroRNA-29b: A Novel Mechanism in Regulation of Liver Fibrosis. Mol Ther 2017;25:205-217.

29 Zheng J, Wu C, Lin Z, Guo Y, Shi L, Dong P, Lu Z, Gao S, Liao Y, Chen B, Yu F: Curcumin up-regulates phosphatase and tensin homologue deleted on chromosome 10 through microRNA-mediated control of DNA methylation--a novel mechanism suppressing liver fibrosis. FEBS J 2014;281:88-103.

-30 Ribon V, Johnson JH, Camp HS, Saltiel AR: Thiazolidinediones and insulin resistance: peroxisome proliferatoractivated receptor gamma activation stimulates expression of the CAP gene. Proc Natl Acad Sci U S A 1998;95:14751-14756. 


\section{Cellular Physiology Cell Physiol Biochem 2018;46:82-92 and Biochemistry Published online:March 23, $2018 \quad \begin{aligned} & \text { DOI 10159/2018 The Author(s). Published by S. Karger AG, Basel } \\ & \text { www.karger.com/cpb }\end{aligned}$ \\ Zheng et al.: MiR-30a Inhibits EMT in HSC Activation by Targeting Snai1}

31 Suzuki T, Mizutani K, Minami A, Nobutani K, Kurita S, Nagino M, Shimono Y, Takai Y: Suppression of the TGF-beta1-induced protein expression of SNAI1 and N-cadherin by miR-199a. Genes Cells 2014;19:667675.

-32 Li WF, Dai H, Ou Q Zuo GQ, Liu CA: Overexpression of microRNA-30a-5p inhibits liver cancer cell proliferation and induces apoptosis by targeting MTDH/PTEN/AKT pathway. Tumour Biol 2016;37:58855895.

-33 Liu X, Ji Q, Zhang C, Liu Y, Liu N, Sui H, Zhou L, Wang S, Li Q: miR-30a acts as a tumor suppressor by doubletargeting COX-2 and BCL9 in H. pylori gastric cancer models. Sci Rep 2017;7:7113.

34 Chung YH, Li SC, Kao YH, Luo HL, Cheng YT, Lin PR, Tai MH, Chiang PH: MiR-30a-5p Inhibits Epithelial-toMesenchymal Transition and Upregulates Expression of Tight Junction Protein Claudin-5 in Human Upper Tract Urothelial Carcinoma Cells. Int J Mol Sci 2017;18

35 Duisters RF, Tijsen AJ, Schroen B, Leenders JJ, Lentink V, van der Made I, Herias V, van Leeuwen RE, Schellings MW, Barenbrug P, Maessen JG, Heymans S, Pinto YM, Creemers EE: miR-133 and miR-30 regulate connective tissue growth factor: implications for a role of microRNAs in myocardial matrix remodeling. Circ Res 2009;104:170-178, 176p following 178.

-36 Peinado H, Olmeda D, Cano A: Snail, Zeb and bHLH factors in tumour progression: an alliance against the epithelial phenotype? Nat Rev Cancer 2007;7:415-428.

37 Cano A, Perez-Moreno MA, Rodrigo I, Locascio A, Blanco MJ, del Barrio MG, Portillo F, Nieto MA: The transcription factor snail controls epithelial-mesenchymal transitions by repressing E-cadherin expression. Nat Cell Biol 2000;2:76-83. 